\title{
Carcinoma adenoide quístico primario de bronquio lobar: Caso clínico*
}

\author{
Drs. LEONIDAS TAPIAS-VARGAS ${ }^{1}$, LUIS FELIPE TAPIAS-VARGAS ${ }^{2}$, LEONIDAS TAPIAS ${ }^{3}$, \\ MARÍA EMMA GARCÍA ${ }^{3}$, JULIO CÉSAR MANTILLA ${ }^{4}$, ERNESTO GARCÍA ${ }^{4}$
}

1 Departamento de Cirugía. Saint Mary's Hospital, Waterbury, CT, USA.

2 Escuela de Medicina. Universidad Industrial de Santander, Bucaramanga, Santander, Colombia.

3 Departamento de Cirugía y Patología, Fundación Oftalmológica de Santander-Clínica Carlos Ardilla Lülle, Floridablanca, Santander, Colombia.

4 Grupo de Investigación en Patología Estructural, Funcional y Clínica. Universidad Industrial de Santander, Bucaramanga, Santander, Colombia.

\begin{abstract}
Primary adenoid cystic carcinoma of the lobar bronchus. Case report

Primary airway adenoid cystic carcinoma is very uncommon. We report a 60 years old male consulting for hemoptysis and dyspnea. A chest CAT scan showed a mass in the right superior lobar bronchus. The patient was subjected to a right superior sleeve lobectomy and the pathological study of the surgical piece revealed an adenoid cystic carcinoma. The patient received adjuvant radiotherapy. Surgery and radiation therapy are the mainstay of treatment for this type of tumors.
\end{abstract}

Key words: Adenoid cystic carcinoma, lobectomy, radiotherapy.

\section{Resumen}

El carcinoma adenoide quístico primario de la vía aérea es una neoplasia muy rara. Reportamos el caso de un paciente de 60 años de edad quien consultó por hemoptisis y disnea de esfuerzo. Una tomografia computarizada del tórax reveló una masa en el bronquio fuente y lobar superior del pulmón derecho. Se realizó una lobectomía superior derecha en manguito. El estudio histopatológico mostró un carcinoma adenoide quístico. Se administró radioterapia adyuvante. La cirugía y la radioterapia son las bases del manejo de este tipo de tumores.

Palabras clave: Carcinoma quístico adenoide, neoplasias pulmonares, cirugía torácica, neumonectomía.

\section{Descripción del caso}

Un hombre de 60 años de edad, consultó en marzo de 2007 por episodios recurrentes de hemoptisis de 2 años de evolución y reciente aparición de dis- nea de esfuerzo. Tenía una historia de hipertensión arterial en manejo con enalapril y de tabaquismo el cual había dejado 35 años atrás. Se solicitaron estudios de esputo con coloración de Zielh-Nielsen los cuales fueron negativos para micobacterias. Su

* Recibido el 25 de Mayo de 2010 y aceptado para publicación el 21 de Julio de 2010.

Correspondencia: Dr. Luis Felipe Tapias-Vargas. Cr 23B \#28-25, La Pera, Floridablanca, Santander, Colombia. fax: 6382822.

ftapias@gmail.com 
curva flujo-volumen mostró una capacidad vital forzada (FVC) de 3,3L y un volumen espiratorio forzado en 1 segundo de 2,65L. Se solicitó una tomografía computarizada (TC) del tórax la cual puso en evidencia una masa en el bronquio fuente derecho (BFD) con signos de neumonitis post-obstructiva (Figura 1). Se extendió el estudio con una broncoscopia flexible, observándose una lesión obstructiva sugestiva de neoplasia en el BFD y en el bronquio lobar superior derecho (BLSD), de la cual se tomó una biopsia. Esta biopsia fue reportada como un tumor carcinoide. Se decidió llevar el paciente a cirugía para la realización de una lobectomía superior derecha en manguito con broncoplastía en abril de 2007. Intraoperatoriamente se notó que la masa se originaba del BLSD y se extendía al BFD y el bron-
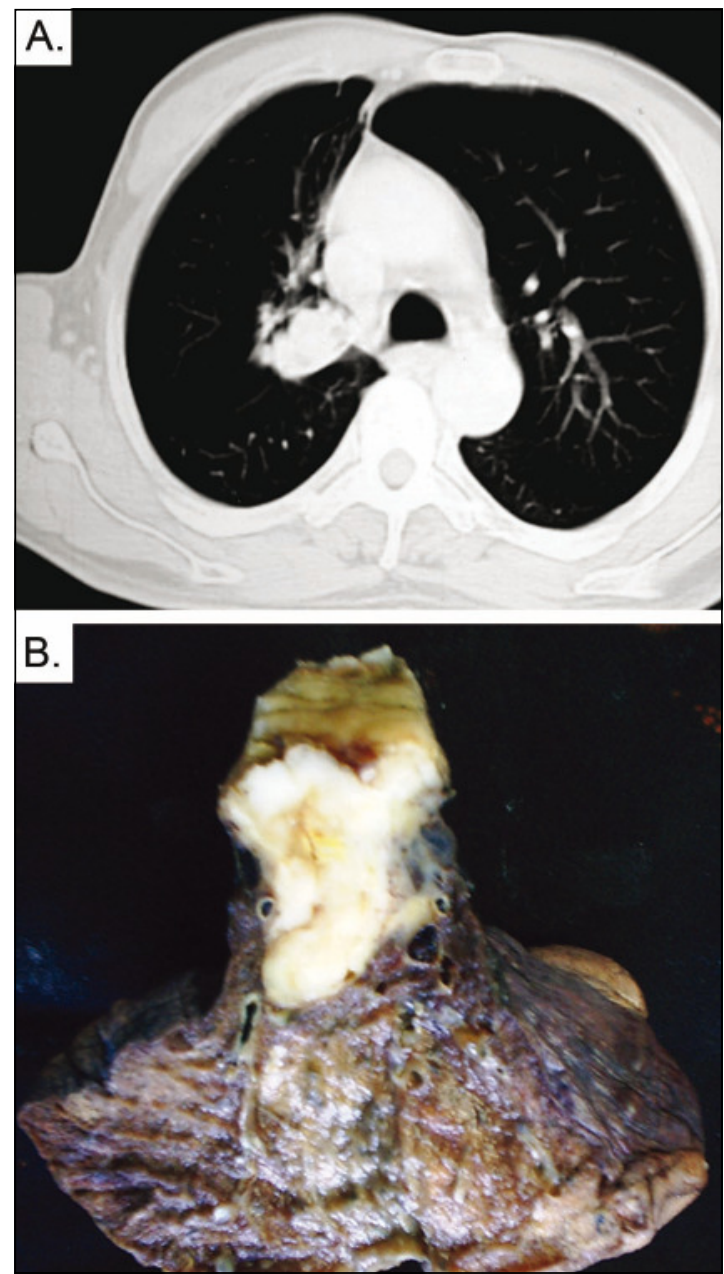

Figura 1. A. TC de tórax preoperatoria mostrando una masa en el BFD. B. Superficie de corte del lóbulo superior derecho mostrando el CAQ. quio intermedio (BI). Se realizó la resección junto con una anastomosis término-terminal entre el BFD y el BI, sin complicaciones. El estudio histopatológico de la pieza quirúrgica ofreció el diagnóstico de carcinoma adenoide quístico bronquial con invasión linfática e infiltración perineural, metastásico a un ganglio peribronquial, con compromiso microscópico de un margen de sección. Las tinciones de inmunohistoquímica fueron positivas para panqueratina, $\alpha 1$-antiquimiotripsina y S100. Una nueva TC en el postoperatorio demostró la integridad y permeabilidad de la anastomosis bronquial. El paciente recibió 61,2 Gy de radioterapia sobre la lesión tumoral hasta julio de 2007. Una nueva TC en diciembre de 2007 mostró tractos fibrosos bilaterales y bronquiectasias relacionadas con la radioterapia. Luego de 2,5 años de seguimiento no hay evidencia de recurrencia o enfermedad metastásica.

\section{Discusión}

Los tumores primarios de la vía aérea son muy raros, estimándose que aparecen 600 a 700 casos al año en Estados Unidos ${ }^{1}$. La gran mayoría de tumores primarios de la tráquea son carcinomas escamocelulares, seguidos de los carcinomas adenoides quísticos (CAQ) representando cada uno el 46\% y el $26 \%$ de las malignidades primarias, respectivamente $^{2}$. El CAQ es un tumor de tipo salivar que ha sido reportado en varias localizaciones anatómicas como las glándulas mamarias, la piel, el útero y el pulmón donde se dice que representa menos del $1 \%$ de las neoplasias malignas primarias. El CAQ de la vía aérea es de crecimiento lento y se origina más frecuentemente en la tráquea, por lo que los $\mathrm{CAQ}$ originarios del bronquio lobar como el que describimos arriba son poco comunes. En el Massachusetts General Hospital, 12 de 78 tumores bronquiales primarios eran derivados de glándulas de la vía aérea, y de éstos, solamente 2 casos fueron $\mathrm{CAQ}^{3}$. Existen dos reportes de la Mayo Clinic que abarcan ambos 50 años de experiencia en tumores glandulares bronquiales; en el primer reporte, $8 \%$ de los tumores bronquiales glandulares fueron $\mathrm{CAQ}$, siendo los tumores carcinoides la gran mayoría con $90 \%$ de los $\operatorname{casos}^{4}$; en el segundo reporte, se recolectaron $20 \mathrm{CAQ}$, haciendo de esa la serie más grande hasta el momento de CAQ bronquiales ${ }^{5}$. En un estudio reciente de 14 CAQ traqueo-bronquiales, 4 casos tenían enfermedad coexistente de la tráquea y bronquios y 2 casos mostraron compromiso bronquial sin infiltración traqueal ${ }^{6}$. Los CAQ de la vía aérea han sido reportados con una distribución por sexo casi igual y han sido encontrados más frecuentemente en personas no fumadoras ${ }^{2,3,6}$. 
El CAQ de la vía aérea se manifiesta clínicamente dependiendo de su localización ${ }^{1}$. Las lesiones traqueales tienen presentaciones insidiosas, debutando con disnea de esfuerzo, sibilancias, estridor, tos, hemoptisis e infecciones pulmonares recurrentes. Las lesiones bronquiales usualmente dan síntomas relacionadas con la función pulmonar comprometida de manera unilateral, neumonía post-obstructiva, atelectasias, consolidaciones o hiperinflación. Generalmente, los pacientes con tumores primarios de la vía aérea, incluyendo el CAQ, pueden haber sido diagnosticados erróneamente con asma o enfermedad pulmonar obstructiva crónica muchas veces; por lo tanto, los casos de asma atípicamente refractaria o de inicio en la edad adulta deben elevar sospecha y llevar a una investigación más profunda. El examen físico no es de ayuda en la mayoría de los casos. El diagnóstico definitivo se logra a través de la visualización directa o indirecta del tumor; los estudios radiológicos (tomografia) y la broncoscopia son las bases para realizar el diagnóstico.

El tratamiento consiste esencialmente en la resección total del tumor. La resección mediante broncoscopia puede dejar atrás una cantidad significativa de tumor, por lo que su papel es paliativo en pacientes que no pueden ser sometidos a cirugía mayor, o temporal, para restablecer el flujo de aire en un escenario de emergencia. La resección completa puede lograrse mediante técnicas "ahorradoras" y "no-ahorradoras" de pulmón. La neumonectomía fue el estándar de tratamiento varios años atrás ${ }^{4}$, sin embargo, hoy en día las resecciones bronquiales y lobectomías en manguito permiten una menor pérdida de parénquima pulmonar con buenos resultados. La radioterapia se usa como tratamiento complementario después de la cirugía ya que con frecuencia se dejan restos microscópicos de tumor en los bordes de sección; es importante anotar que el CAQ ha demostrado ser bastante radiosensible. A pesar de esto, la resección completa debe buscarse siempre que sea posible, debido a que el CAQ recurre fácilmente y el pronóstico puede verse afectado.

El pronóstico depende principalmente del tratamiento instaurado. Los tumores no resecados tienen tasas de sobrevida a 5 y 10 años de $40 \%$ y $0 \%$, res- pectivamente ${ }^{7}$. Los tumores resecados tienen tasas de sobrevida a 5 y 10 años de $91 \%$ y $76 \%$, respectivamente. Sin embargo, en un reporte que acumulaba 32 años de experiencia, se alcanzó una sobrevida a 10 años de $69 \%$ con la resección completa, contra sólo $30 \%$ cuando quedaba enfermedad microscópica residual $^{8}$. Incluso si se realiza una resección incompleta, la sobrevida es mucho mejor que la alcanzada sin cirugía; se ha documentado la sobrevida hasta por 22 años luego de la resección incompleta 9 .

\section{Referencias}

1. Wood DE. Management of malignant tracheobronchial obstruction. Surg Clin North Am. 2002;82:621-42.

2. Webb BD, Walsh GL, Roberts DB, Sturgis EM. Primary tracheal malignant neoplasms: the University of Texas MD Anderson Cancer Center experience. J Am Coll Surg. 2006;202:237-46.

3. Gaissert HA, Mark EJ. Tracheobronchial gland tumors. Cancer Control 2006;13:286-94.

4. Payne WS, Ellis FH Jr, Woolner LB, Moersch HJ. The surgical treatment of cylindroma (adenoid cystic carcinoma) and muco-epidermoid tumors of the bronchus. J Thorac Cardiovasc Surg. 1959;38:709-26.

5. Conlan AA, Payne WS, Woolner LB, Sanderson DR. Adenoid cystic carcinoma (cylindroma) and mucoepidermoid carcinoma of the bronchus. Factors affecting survival. J Thorac Cardiovasc Surg. 1978;76:369-77.

6. Albers E, Lawrie T, Harrell JH, Yi ES. Tracheobronchial adenoid cystic carcinoma: a clinicopathologic study of 14 cases. Chest 2004;125:1160-5.

7. Kanematsu T, Yohena T, Uehara T, Sakiyakma S, Kenzaki K, Kondo K, et al. Treatment outcome of resected and nonresected primary adenoid cystic carcinoma of the lung. Ann Thorac Cardiovasc Surg. 2002;8:74-7.

8. Maziak DE, Todd TR, Keshavjee SH, Winton TL, Van Nostrand P, Pearson FG. Adenoid cystic carcinoma of the airway: thirty-two-year experience. J Thorac Cardiovasc Surg. 1996;112:1522-31.

9. Schoenfeld N, Rahn W, Loddenkemper R. Twenty two year survival after incomplete resection of advanced adenoid cystic bronchogenic carcinoma. Eur Respir J. 1996;9:1560-1. 